\title{
Development and assessment of a medium-range real-time kinematic GPS algorithm using an ionospheric information filter
}

\author{
Ming Yang ${ }^{1}$, Chin-Hsien Tang ${ }^{1}$, and Ting-To $\mathrm{Yu}^{2}$ \\ ${ }^{1}$ Department of Surveying Engineering, National Cheng Kung University, Tainan, Taiwan \\ ${ }^{2}$ Satellite Geoinformatics Research Center, National Cheng Kung University, Tainan, Taiwan
}

(Received January 31, 2000; Revised August 28, 2000; Accepted August 28, 2000)

\begin{abstract}
The key requirement of centimeter-level real-time kinematic (RTK) positioning using the Global Positioning System (GPS) relies on the ability to fast and accurately determine the ambiguities of carrier-phase observations to their inherent integer values. In addition, the identification must be completed on the fly since the remote receiver is constantly in motion. The Kalman filter-based algorithm described in this paper uses an ionospheric information filter to perform on-the-fly phase ambiguity resolution for high precision RTK applications. Experiments based on 16 independent test baselines ranging from $10-50 \mathrm{~km}$ in length indicate that the algorithm can reliably achieve centimeter-level positioning accuracy, provided that a small enough threshold value for ambiguity identification is pre-defined and that sufficient geometry change in the GPS constellation is observed. Experimental results also show that the convergence (initialization) time for ambiguity resolution is linearly proportional to instantaneous baseline length, and the slope of the regression line increases with tighter ambiguity identification criteria.
\end{abstract}

\section{Introduction}

Precise determination of double-differenced GPS phase ambiguities on the fly is the most important task for high precision kinematic applications (Yang et al., 1994; Goad and Yang, 1997; Han, 1997; Teunissen, 1997; Mohamed and Schwarz, 1999; Wang, 2000). Today, most GPS-based high precision RTK surveys have a limitation on the distance between the rover and the base receivers, the so-called baseline length. Rarely will one find routine centimeter-level RTK applications over longer baselines. The main obstacles in longer-range real-time ambiguity resolution are twofoldresidual orbital error in the GPS broadcast ephemeris, and residual signal delay caused by the atmosphere including the ionosphere and the troposphere. In general, the resolution of GPS phase ambiguity is simpler and straightforward for short-range applications, as the differential influences on GPS observables caused by the GPS broadcast orbits and the atmosphere can be canceled by the sufficient similarities at the two ends. However, for longer-range baselines, the above assumption is no longer valid, and consequently the systematic influences must be carefully modeled.

In this paper, the term "medium-range" thus refers to the assumption that the double-differenced GPS phase observations are sufficiently insensitive to the relatively smaller residual effects, namely, the GPS orbital error and the tropospheric refraction. Hence, only the differential ionospheric influence needs to be effectively modeled for successful resolution of phase integer ambiguity. This is particularly important to engineers and geodesists who wish to perform

Copy right $(\mathrm{C}$ The Society of Geomagnetism and Earth, Planetary and Space Sciences (SGEPSS); The Seismological Society of Japan; The Volcanological Society of Japan; The Geodetic Society of Japan; The Japanese Society for Planetary Sciences. high precision RTK surveys in areas located in the so-called equatorial anomaly region. Taiwan for example, at $10-14^{\circ} \mathrm{N}$ geomagnetic, is situated in the region (Huang et al., 1989). Irregularities of the ionosphere mainly occur in the equatorial anomaly region that is in the shape of a belt surrounding the earth's geomagnetic equator, as well as in the polar auroral zones; however, a very high electron content only occurs in the equatorial region (Seeber, 1993, p. 306). As a result, noticeable residual ionospheric signals can be seen in doubledifferenced phase observations even over short baselines in this area (ibid.).

It has long been recognized that using multiple reference stations improves differential GPS positioning results. Such a multiple-reference configuration is generally referred to as wide-area differential GPS (WADGPS), mainly implemented for single frequency code or phase-smoothed code positioning with varying levels of accuracy from a few decimeters to a few meters (Ashkenazi et al., 1993; Bertiger et al., 1998). In recent years, researchers started to take advantage of the multi-reference configuration and successfully achieved fast ambiguity resolution for centimeter-level RTK positioning using dual frequency carrier-phase measurements. For instance, Han (1997), Raquet and Lachapelle (2000) independently developed methods to process dual frequency GPS data and perform interpolation of differential ionospheric delays using a network of reference stations.

Nevertheless, due to the cost factor, the majority of today's high precision RTK positioning projects and commercial products still operate on the so-called single-reference mode, i.e., only one reference station is used in the communication link and as a result, no interpolation of differential influences can be performed. This means that in the single-reference mode, the only direct source that contains 
information about the differential ionospheric signals is the GPS dual frequency phase and pseudorange observations. To model the differential ionospheric refraction for a single baseline, Goad and Yang (1997) proposed an approach based on a smoothing technique that operates on single-differenced GPS measurements. In this approach, all available GPS data are combined by a smoother for the estimation of a group of time-varying ionospheric parameters. For real-time applications, however, such a smoother-based technique is no longer effective. Moreover, since computational time must be taken into consideration in a real-time situation, it is advantageous to reduce the number of estimated parameters in the state vector to its minimum. Consequently, the processing of single-differenced measurements is not optimal for RTK implementation.

In this paper, a Kalman filter-based algorithm with a realtime application capability is used to combine doubledifferenced phase and pseudorange data, and to take advantage of the stochastic structure in the ionospheric signal. An integer transformation procedure is also incorporated into the algorithm to decorrelate the float ambiguity estimates for optimal identification of their correct integer values.

As both the accuracy level and time factor play crucial roles in high precision RTK applications, the purpose of this paper is to assess, with independent test baselines of various lengths, the algorithm's ability to achieve centimeter-level kinematic positioning, and to investigate the relationship between convergence time, instantaneous baseline distance, and threshold values for ambiguity identification.

\section{The Filter}

The GPS dual frequency double-differenced observations measured between receivers $i$ and $j$ (subscript), and satellites $k$ and $l$ (superscript) are given as follows (Goad and Yang, 1997; Yang and Lo, 2000):

$$
\begin{aligned}
& \Phi_{i j, 1}^{k l}=\rho_{i j}^{k l}-\frac{I_{i j}^{k l}}{f_{1}^{2}}+T_{i j}^{k l}+\lambda_{1} N_{i j, 1}^{k l}+\epsilon_{i j, 1}^{k l}, \\
& \Phi_{i j, 2}^{k l}=\rho_{i j}^{k l}-\frac{I_{i j}^{k l}}{f_{2}^{2}}+T_{i j}^{k l}+\lambda_{2} N_{i j, 2}^{k l}+\epsilon_{i j, 2}^{k l}, \\
& P_{i j, 1}^{k l}=\rho_{i j}^{k l}+\frac{I_{i j}^{k l}}{f_{1}^{2}}+T_{i j}^{k l}+e_{i j, 1}^{k l}, \\
& P_{i j, 2}^{k l}=\rho_{i j}^{k l}+\frac{I_{i j}^{k l}}{f_{2}^{2}}+T_{i j}^{k l}+e_{i j, 2}^{k l},
\end{aligned}
$$

where $\Phi_{1}, \Phi_{2}, P_{1}, P_{2}$ are the phase ranges and pseudoranges measured at the $\mathrm{L}_{1}$ and $\mathrm{L}_{2}$ frequencies, respectively. The term $\rho$ is the geometric distance between the two satellite antennas and the two receiver antennas while $I / f^{2}$ stands for the first-order atmospheric ionosphere refraction with group delays associated with the pseudoranges and phase advances associated with the phases. The wavelengths of the $\mathrm{L}_{1}$ and $\mathrm{L}_{2}$ phases are $\lambda_{1} \approx 19 \mathrm{~cm}$ and $\lambda_{2} \approx 24 \mathrm{~cm}$, respectively. The measurement noise is characterized by the error terms $\epsilon$ and $e$. The integer ambiguities associated with the $\mathrm{L}_{1}$ and $\mathrm{L}_{2}$ phases are denoted as $N_{1}$ and $N_{2}$, respectively.

The tropospheric effect $T$ can be estimated together with surface meteorological data by some tropospheric correction models, for instance, those of Hopfield (1969) and Saastamoinen (1972). Despite the differences between the various models, when the elevation angle is above 20 degrees, different models give very similar estimates of the tropospheric refraction (Solheim, 1993).

The effect of ionosphere on GPS measurements is generally regarded as a time-dependent signal that continuously changes in time as the electron density varies with time and location in the ionosphere (Klobuchar, 1991). In addition to the dependence on time, the effect of differential ionosphere is also a function of baseline length (Dong and Bock, 1989). When the two receivers are close to each other, the differential ionospheric refraction is greatly reduced due to cancellation; however, as the baseline length is increased, the reduction becomes less significant (Yang et al., 1994).

The model for estimating the phase ambiguities is given in a Kalman filter form. At a given epoch $t_{n}$, the linearized observation equation is formulated as follows:

$$
Z_{n}=H_{n} \cdot x_{n}+\epsilon_{n}
$$

where $Z_{n}$ is the observation vector comprising the four measurements, and a zero a priori observation of the doubledifferenced ionosphere. The error vector is $\epsilon$ and $H$ is the design matrix. Here the state vector is defined as

$$
x_{n}=\left[\Delta x_{j} \Delta y_{j} \Delta z_{j} \frac{I}{f_{1}^{2}} N_{1} N_{1}-N_{2}\right]^{T}
$$

where $\Delta x_{j}, \Delta y_{j}, \Delta z_{j}$ represent the corrections made to the reference position of the moving receiver $j$ at epoch $t_{n}$. The term $I / f_{1}^{2}$ stands for the $\mathrm{L}_{1}$ double-differenced ionospheric refraction associated with the measurements. The doubledifferenced integer ambiguities of the $\mathrm{L}_{1}$ and widelane $\left(\mathrm{L}_{1}-\right.$ $\mathrm{L}_{2}$ ) phases are denoted as $N_{1}$ and $N_{1}-N_{2}$, respectively. The choice of the widelane phase is mainly for its longer wavelength.

The prediction $(-)$ of the state vector at the next epoch $n+1$ is derived from the update $(+)$ of epoch $n$ as follows (Gelb, 1979, p. 110):

$$
x_{n+1}(-)=\phi_{n} x_{n}(+)+w_{n}, \quad w_{n} \sim N\left(0, Q_{n}\right) .
$$

In this study we define the state transition matrix $\phi_{n}$ and corresponding covariance matrix $Q_{n}$ as

$$
\begin{aligned}
\phi_{n} & =\left[\begin{array}{llllll}
1 & 0 & 0 & 0 & 0 & 0 \\
0 & 1 & 0 & 0 & 0 & 0 \\
0 & 0 & 1 & 0 & 0 & 0 \\
0 & 0 & 0 & p_{\text {ion }} & 0 & 0 \\
0 & 0 & 0 & 0 & 1 & 0 \\
0 & 0 & 0 & 0 & 0 & 1
\end{array}\right], \\
Q_{n} & =\left[\begin{array}{cccccc}
\infty & 0 & 0 & 0 & 0 & 0 \\
0 & \infty & 0 & 0 & 0 & 0 \\
0 & 0 & \infty & 0 & 0 & 0 \\
0 & 0 & 0 & \sigma_{\text {Wion }}^{2} & 0 & 0 \\
0 & 0 & 0 & 0 & 0 & 0 \\
0 & 0 & 0 & 0 & 0 & 0
\end{array}\right]
\end{aligned}
$$


where $p_{\text {ion }}$ represents the prediction operator used to predict the double-differenced ionospheric delay from one epoch to the next, over a time interval $\tau$ and a baseline length change $\delta$ while the corresponding variance of the prediction error is denoted as $\sigma_{\text {Wion }}^{2}$. Their values are given by Goad and Yang (1997) as

$$
\begin{aligned}
& p_{\text {ion }}=e^{-(|\tau| / T+|\delta| / D)}, \\
& \sigma_{\text {Wion }}^{2}=\sigma_{\infty}^{2}\left(1-e^{-2(|\tau| / T+|\delta| / D)}\right)
\end{aligned}
$$

where $T=64 \mathrm{~min}$ stands for the first-order correlation time for the double-differenced ionosphere, and $D=1500 \mathrm{~km}$ represents the first-order correlation distance. The variance of the double-differenced ionosphere with $\tau$ and $\delta$ as the two independent variables is $\sigma_{\infty}^{2}=2.0 \mathrm{~m}^{2}$.

The remaining diagonal elements of the state transition matrix $\phi_{n}$ are set to one, indicating that in the filter the predicted values for the moving receiver's position and phase ambiguities are unchanged over time. Obviously this assumption cannot be true. Therefore, a very large number is assigned to each of the first three diagonal elements of the corresponding covariance matrix $Q_{n}$, in order to effectively neglect any previous information about the moving receiver's position. The last two diagonal elements of $Q_{n}$ are set to zero when continuous tracking is maintained; otherwise, they are set to very large numbers when estimation of new ambiguities is requested.

\section{Fixing Integer Ambiguities}

The most intuitive way to constrain float ambiguity estimates to integer values is to round the estimates to their respective nearest integers. However, this method is optimal only if the estimates have reached a steady state with high precision, and the correlation between the estimates is low. In the algorithm, the integer transformation concept proposed by Teunissen $(1995,1997)$ that minimizes both the correlation between the integer ambiguities and the elongation of the error ellipsoid is used to decorrelate the normally highly correlated GPS phase ambiguities. Suppose that two doubledifferenced phase ambiguity estimates and their associated covariance matrix are available from the filter as

$$
\hat{a}=\left[\begin{array}{l}
\hat{a}_{1} \\
\hat{a}_{2}
\end{array}\right], \quad Q_{\hat{a}}=\left[\begin{array}{ll}
\sigma_{\hat{a}(1,1)} & \sigma_{\hat{a}(1,2)} \\
\sigma_{\hat{a}(2,1)} & \sigma_{\hat{a}(2,2)}
\end{array}\right] .
$$

The integer transformation process is performed as (Teunissen, 1995)

$$
\begin{aligned}
{\left[\begin{array}{l}
\hat{a}_{1^{\prime}} \\
\hat{a}_{2^{\prime}}
\end{array}\right] } & =\left[\begin{array}{c}
1-\left\{\sigma_{\hat{a}\left(1,2^{\prime}\right)} \sigma_{\hat{a}\left(2^{\prime}, 2^{\prime}\right)}^{-1}\right\} \\
0 \\
1
\end{array}\right]\left[\begin{array}{cc}
1 & 0 \\
-\left\{\sigma_{\hat{a}(2,1)} \sigma_{\hat{a}(1,1)}^{-1}\right\} & 1
\end{array}\right]\left[\begin{array}{l}
\hat{a}_{1} \\
\hat{a}_{2}
\end{array}\right] \\
& =Z^{T}\left[\begin{array}{l}
\hat{a}_{1} \\
\hat{a}_{2}
\end{array}\right]
\end{aligned}
$$

where the symbol \{\} stands for rounding to the nearest integer. The associated covariance matrix is consequently obtained as

$$
Q_{\hat{a}}^{\prime}=Z^{T}\left[\begin{array}{ll}
\sigma_{\hat{a}(1,1)} & \sigma_{\hat{a}(1,2)} \\
\sigma_{\hat{a}(2,1)} & \sigma_{\hat{a}(2,2)}
\end{array}\right] Z=\left[\begin{array}{cc}
\sigma_{\hat{a}^{\prime}(1,1)} & \sigma_{\hat{a}^{\prime}(1,2)} \\
\sigma_{\hat{a}^{\prime}(2,1)} & \sigma_{\hat{a}^{\prime}(2,2)}
\end{array}\right]
$$

The integer transformation procedure generates a linear combination of the original integer ambiguities to achieve the goal of decorrelation (Teunissen, 1995). To successfully resolve the correct integer values of the transformed (and decorrelated) ambiguities, two conditions need to be fulfilled at once: the float estimates should reach a steady state with high precision, and the correlations between the estimates should be sufficiently low. In general, if the integer transformation procedure is successful, the above conditions can be both fulfilled (Peiliang Xu, 2000, personal communication). In this study, a simplified measure is adopted to determine the ambiguity integers. If the associated standard deviations of the transformed ambiguity estimates are smaller than a pre-defined threshold value $\kappa$

$$
\sqrt{\sigma_{\hat{a}^{\prime}}} \leq \kappa
$$

then the transformed float ambiguity estimates are rounded (and constrained) to their nearest integers, and the state vector and the covariance matrix of the filter are updated accordingly.

To extend from the two-dimensional case to a more general multi-dimensional case, the integer transformation procedure is repeated and applied to all possible ambiguity pairs in the state vector. After the resolution of phase integer ambiguity, precise kinematic positioning can be continuously performed.

\section{Test Results}

\subsection{Test data description}

To investigate the applicability of the proposed algorithm for resolution of the phase ambiguity on the fly, 16 independent GPS baselines ranging from $10-50 \mathrm{~km}$ in length were studied. About $40 \mathrm{~min}$ of dual frequency test data were collected between January and April 1997, with a sampling interval of 15 seconds and a satellite elevation cutoff angle of 10 degrees. The test data were in fact static baselines and their "true" static baseline solutions were obtained from off-line data processing using the GPSurvey 2.0 software (Trimble Navigation, Sunnyvale, CA, U.S.A.), with precise GPS ephemeris from the International GPS Service (Neilan et al., 1997; Beutler et al., 1999). Figure 1 displays the spatial distribution of those baselines, which covered various regions of the island of Taiwan, including the central mountainous areas with elevations greater than $2 \mathrm{~km}$ as well as low-elevation areas near the east and west coastlines.

\subsection{Result analyses}

The objective of the experiment was to examine the performance of the proposed algorithm. Three different threshold values, $\kappa=0.15,0.20$, and 0.30 cycles, for the determination of phase ambiguity integer were tested in the experiment. To simulate the real-time scenario, GPS broadcast ephemeris was used.

The accuracy of the kinematic positions is represented by the RMS values between the kinematic positions obtained from the state vector of the filter after the resolution of integer ambiguity, and the "true" baseline solutions acquired offline. Figure 2 illustrates the horizontal and vertical RMS values for the three criteria. For the two tighter threshold values, 0.15 and 0.20 cycles, it is clear that the ambiguity integers are accurately determined, since all the horizontal 


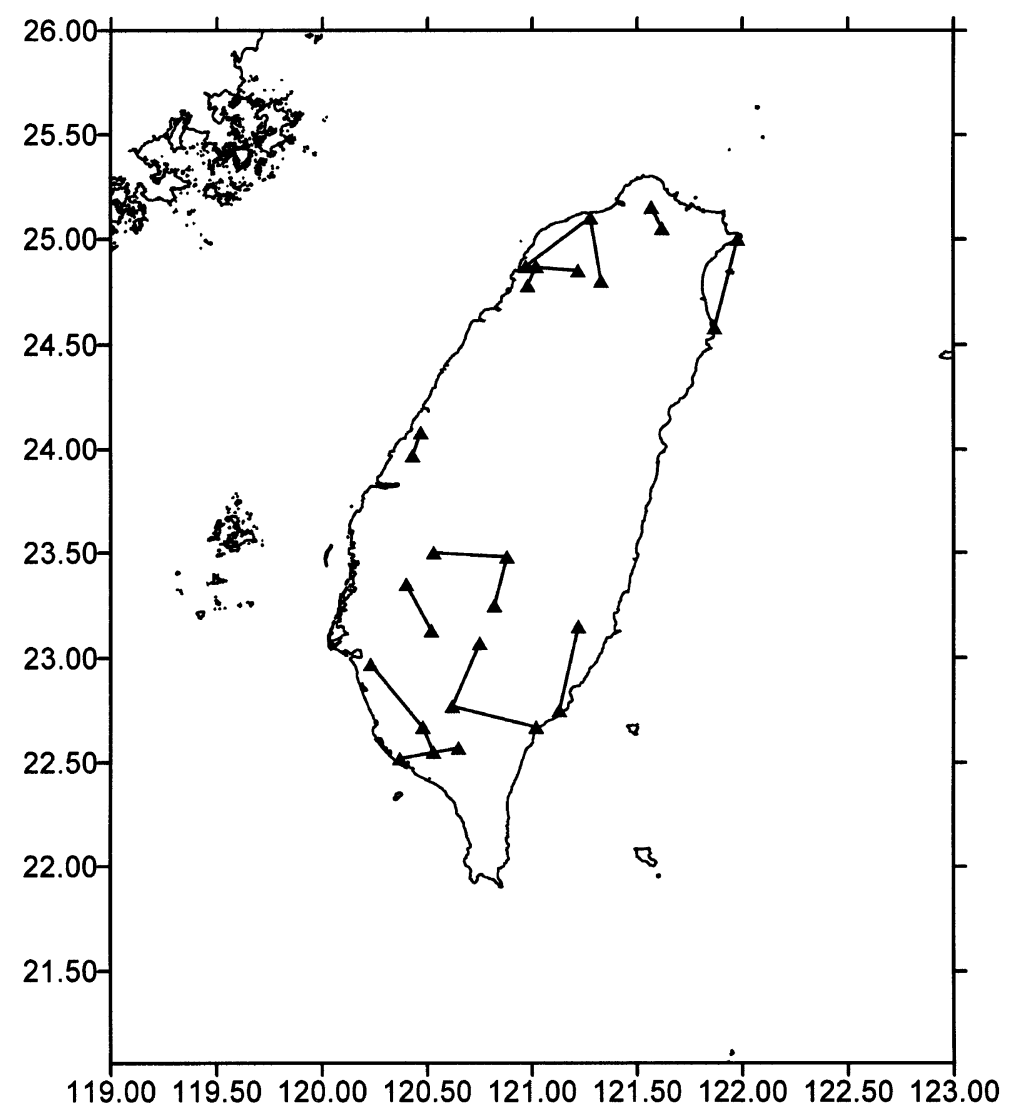

Fig. 1. Distribution of test baselines.
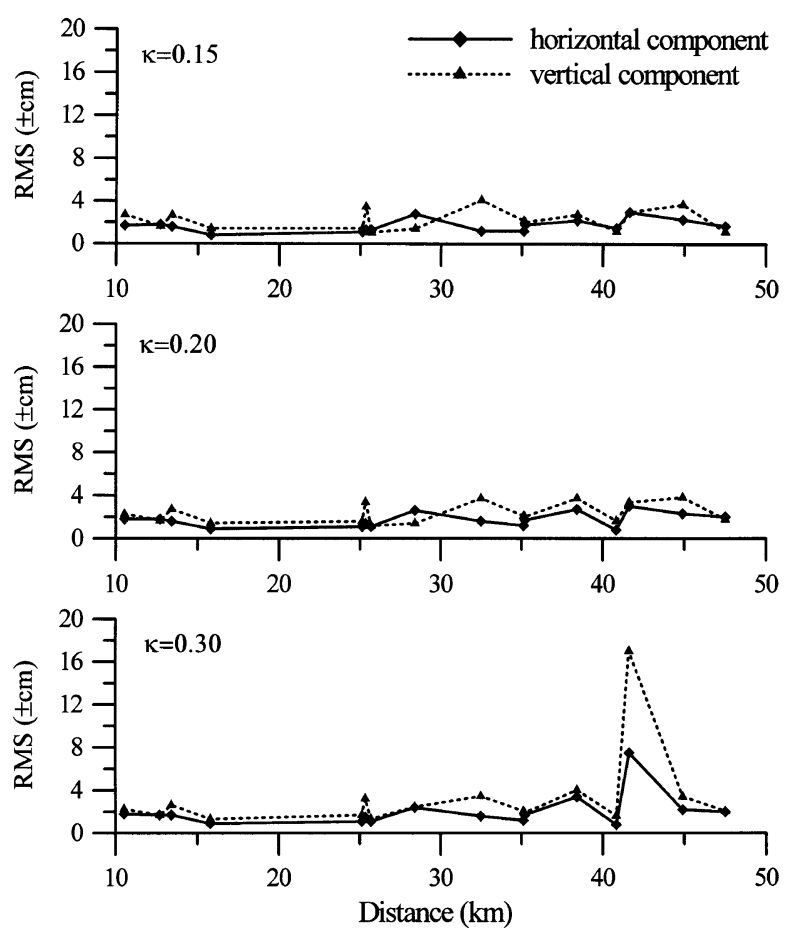

Fig. 2. RMS values vs. baseline distance for various threshold values.
RMS values are within $\pm 3 \mathrm{~cm}$, and all the vertical RMS values are within $\pm 4.2 \mathrm{~cm}$. The length of the baseline only has a small effect on the accuracy level within the distance range of the test baselines. As the baseline distance becomes longer, the RMS values in general only slightly increase. For the loosest threshold value, 0.30 cycles, however, wrong integer values of phase ambiguities are fixed for one of the baselines (a 43-km line), as the corresponding RMS values are as large as $\pm 17.1 \mathrm{~cm}$ vertically, and $\pm 7.5 \mathrm{~cm}$ horizontally. After excluding the incorrect line, similar accuracy estimates to the cases of the two tighter threshold values are obtained.

Figure 3 displays the time (number of epochs) needed for the algorithm to fix integer ambiguity for the three criteria. It is apparent that in all three cases, more time is needed for longer baselines, and the relationship resembles straight lines. Moreover, as expected, the slope of the regression lines increases with tighter threshold values.

To demonstrate the filter's ability to recover the doubledifferenced ionosphere, the filtered estimates and the "true" ionospheric effects obtained from the geometry-free linear combination of the phase data with fixed ambiguities are compared. Figure 4 shows the double-differenced ionospheric signals of two satellite pairs, SV1-6 and SV1-22, for a 47-km line. The integer ambiguity resolution is completed at the 80th epoch for the threshold value of 0.20 cycles. It can be observed that the actual double-differenced ionospheric influence reaches as large as $7 \mathrm{~cm}$ in magnitude, which underscores the argument that the residual ionospheric effects cannot be ignored in the model. Despite the large differ- 


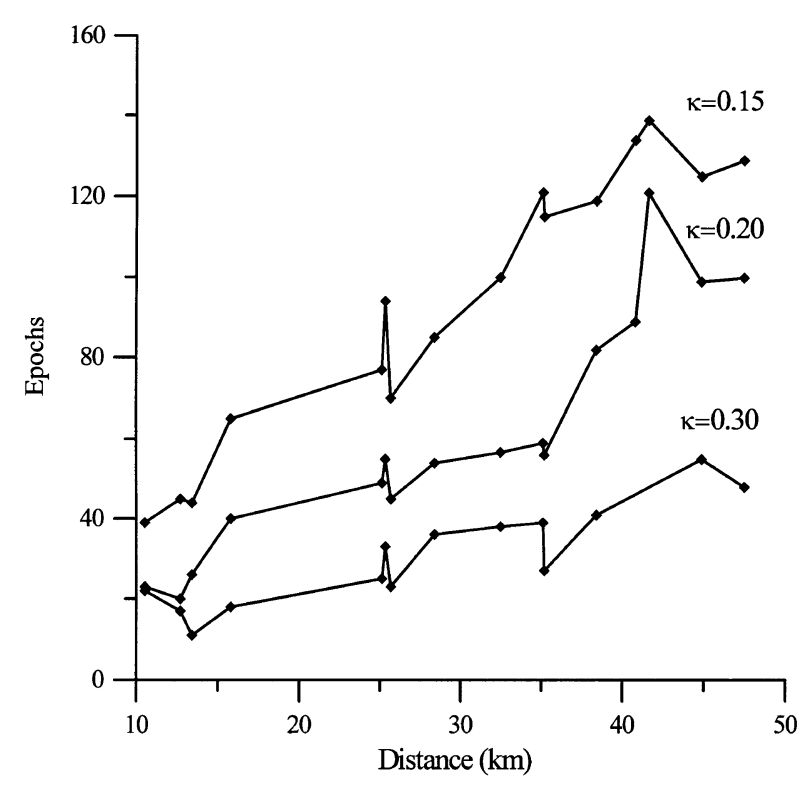

Fig. 3. Number of epochs to resolve integer ambiguity vs. baseline distance.
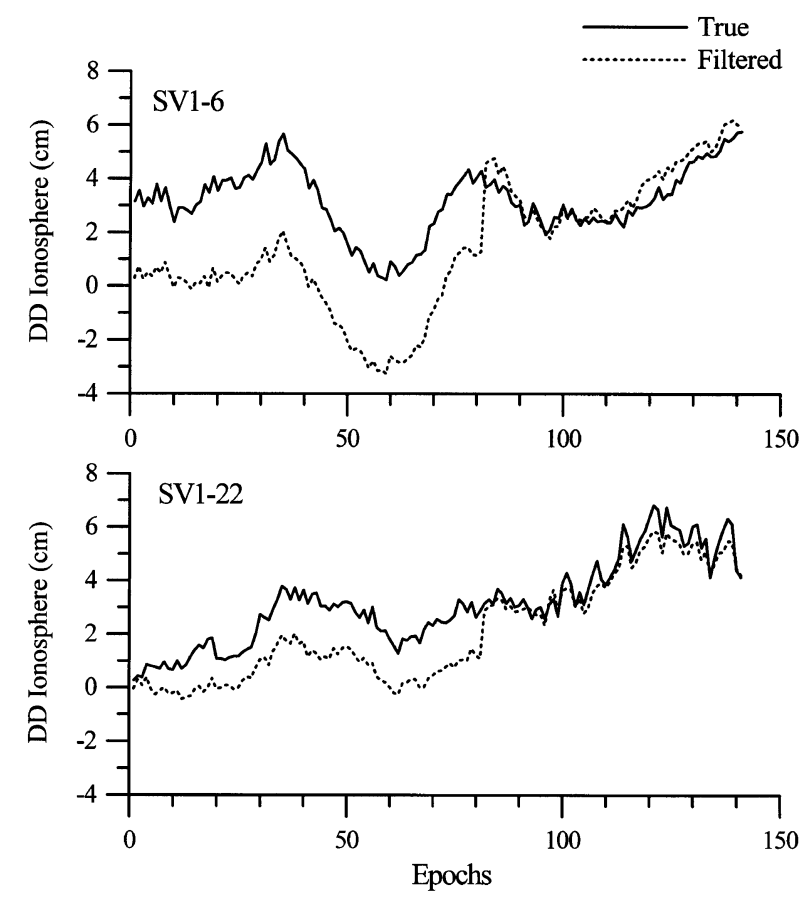

Fig. 4. True and filtered double-differenced ionospheric effects for a 47-km line.

ences in the beginning of the filtering process, the filtered ionospheric estimate gradually approaches the true value as the filter steadily converges. After the resolution of integer ambiguity, the two quantities stay close to each other within $1 \mathrm{~cm}$, i.e., about one twentieth of the wavelengths of the $\mathrm{L}_{1}$ and $\mathrm{L}_{2}$ phases.

\section{Discussion and Conclusion}

At present, most high precision RTK geodetic surveys still operate on the single-reference mode where only one base station is used in the communication link. Since no interpolation of differential ionospheric influences can be performed in the single-reference mode, instantaneous ambiguity resolution in real time becomes very difficult over baselines where the differential ionospheric effect cannot be assumed to be zero. As a result, it is important not only to devise new algorithms for single-baseline RTK ambiguity resolution, but also to investigate the performance of the algorithms from a variety of perspectives - the achievable positioning accuracy, the choice of ambiguity resolution criterion, the convergence (initialization) time, and the relationship between the baseline length and the above factors.

In this paper, an algorithm for medium-range RTK GPS positioning is used to resolve phase ambiguities over test baselines ranging from $10-50 \mathrm{~km}$ in length. The algorithm is combined with an ionospheric information filter and an integer transformation procedure for optimal phase ambiguity resolution.

The use of the algorithm for performing ambiguity resolution yielded varying results, depending on the adopted threshold values for ambiguity identification and the instantaneous baseline distances. From analyses of test results obtained from 16 independent GPS baselines, correct integer ambiguity resolution along with high precision real-time kinematic positions can be reliably obtained, so long as a small enough threshold value for ambiguity identification is pre-defined and sufficient geometry change in the GPS constellation is accumulated over time. Naturally, longer convergence time is needed for smaller threshold values. As the baseline distance increases, so does the required convergence time, and the relationship resembles a straight line whose slope increases with tighter threshold values.

Acknowledgments. This research is supported in part by the National Science Council, R.O.C., under grant NSC 89-2211-E-006127. The authors specially thank Dr. Peiliang Xu and Dr. Gang $\mathrm{Lu}$ for their important suggestions and constructive criticism that greatly improved the quality of the paper.

\section{References}

Ashkenazi, V., C. J. Hill, W. Y. Ochieng, and J. Nagle, Wide-area differential GPS: a performance study, Navigation, 40, 297-319, 1993.

Bertiger, W. I., Y. E. Bar-Sever, B. J. Haines, B. A. Iijima, S. M. Lichten, U. J. Lindqwister, A. J. Mannucci, R. J. Muellerschoen, T. N. Munson, A. W. Moore, L. J. Romans, B. D. Wilson, S. C. Wu, T. P. Yunck, G. Piesinger, and M. Whitehead, A real-time wide area differential GPS system, Navigation, 44, 433-447, 1998.

Beutler, G., M. Rothacher, S. Schaer, T. Springer, J. Kouba, and R. E. Neilan, The international GPS service (IGS): an interdisciplinary service in support of earth sciences, Adv. Space Res., 23, 631-653, 1999.

Dong, D. and Y. Bock, Global positioning system network analysis with phase ambiguity resolution applied to crustal deformation studies in California, J. Geophys. Res., 94, 3949-3966, 1989.

Gelb, A., Applied Optimal Estimation, 370 pp., MIT Press, Cambridge, MA, U.S.A., 1979.

Goad, C. C. and M. Yang, A new approach to precision airborne GPS positioning for photogrammetry, Photogrammetric Engineering and Remote Sensing, 63, 1067-1077, 1997.

Han, S., Carrier Phase-based Long-range GPS Kinematic Positioning, Ph.D. Thesis, 185 pp., School of Geomatic Engineering, The University of New South Wales, Sydney, Australia, 1997.

Hopfield, H. S., Two-quartic tropospheric refractivity profile for correcting satellite data, J. Geophy. Res., 74, 4487-4499, 1969.

Huang, Y. N., K. Cheng, and S. W. Chen, On the equatorial anomaly of the ionospheric total electron content near the northern anomaly crest region, J. Geophys. Res., 94, 13515-13525, 1989. 
Klobuchar, J. A., Ionospheric effect on GPS, GPS World, 2, 48-51, 1991. Mohamed, A. H. and K. P. Schwarz, A simple and economical algorithm for GPS ambiguity resolution on the fly using a whitening filter, Navigation, 45, 221-231, 1999.

Neilan, R. E., J. F. Zumberge, G. Beulter, J. Kouba, The international GPS service: a global resource for GPS applications and research, Proc. ION GPS-97, 883-889, 1997.

Raquet, J. and G. Lachapelle, Development and testing of a kinematic carrier-phase ambiguity resolution method using a reference receiver network, Navigation, 46, 283-295, 2000.

Saastamoinen, I. I., Contribution to the theory of atmospheric refraction, Bulletin Geodesique, 107, 13-34, 1973.

Seeber, G., Satellite Geodesy, 531 pp., Walter de Gruyter, Berlin, Germany, 1993.

Solheim, F. S., Use of pointed water vapor radiometer observations to improve vertical GPS surveying accuracy, Ph.D. Dissertation, Department of Physics, University of Colorado, Boulder, CO, U.S.A., 1993.
Teunissen, P. J. G., The invertible GPS ambiguity transformations, Manuscripta Geodaetica, 20, 489-497, 1995.

Teunissen, P. J. G., The geometry-free GPS ambiguity search space with a weighted ionosphere, J. Geod., 71, 370-383, 1997.

Yang, M. and C. F. Lo, Real-time kinematic GPS positioning for centimeter level ocean surface monitoring, Proc. Natl. Sci. Counc. ROC(A), 24, 79 85, 2000.

Yang, M., C. C. Goad, and B. Schaffrin, Real-time on-the-fly ambiguity resolution over short baselines in the presence of anti-spoofing, Proc. 7 th International Technical Meeting of the Satellite Division of the ION, 519-525, 1994.

Wang, J., Stochastic modeling for real-time kinematic GPS/GLONASS positioning, Navigation, 46, 297-305, 2000.

M. Yang (e-mail: myang@mail.ncku.edu.tw), C.-H. Tang, and T.-T. Yu 